\title{
Development of cardanol-bonded cellulose thermoplastics: high productivity achieved in two-step heterogeneous process
}

\author{
Kiyohiko Toyama $\cdot$ Makoto Soyama \\ Shukichi Tanaka $\cdot$ Masatoshi Iji
}

Received: 28 November 2014/ Accepted: 7 March 2015/Published online: 15 March 2015

(C) The Author(s) 2015. This article is published with open access at Springerlink.com

\begin{abstract}
We developed a heterogeneous process with low energy consumption to synthesize novel high-strength and heat-resistant cellulose-based bioplastics: cellulose esters bonded with a short chain component (acetic acid) and a long one (3-pentadecylphenoxy acetic acid; PAA), which is a derivative of cardanol, extracted from cashew nut shells. Although we recently showed that PAA-bonded cellulose acetates (cardanol-bonded cellulose resins) exhibit highly practical properties for durable products, they were synthesized in a homogeneous process, which requires large quantities of poor solvents to isolate the resins. In the developed two-step heterogeneous process, the cardanol-bonded cellulose resins are produced without poor solvents. In the first step, cellulose is esterified with limited amounts of the long and short chain components using a selected reaction solvent and catalyst. The resultant intermediate products are adequately swollen in the solvent then efficiently recovered by filtration. In the second step, the short chain component is additionally bonded to attain good thermoplasticity of the final products, which are readily recovered only by distilling a solvent and the remaining short chain component. The solvent usage was reduced by approximately $90 \%$ compared with a homogeneous process, which leads to a large
\end{abstract}

K. Toyama $(\bowtie) \cdot$ M. Soyama $\cdot$ S. Tanaka $\cdot$ M. Iji Smart Energy Research Laboratories, NEC Corporation, 34, Miyukigaoka, Tsukuba, Ibaraki 305-8501, Japan e-mail: k-toyama@bq.jp.nec.com reduction in energy. The produced cardanol-bonded cellulose resins exhibited higher thermoplasticity, elastic modulus, water resistivity, and close heat resistivity compared to that of a homogeneous process. Furthermore, the mechanical and thermal characteristics of the resins were greatly improved by adding a specific polyester, polybutylene succinate adipate, and glass fiber, reaching high-level target properties for durable products.

Keywords Bioplastic - Cellulose $\cdot$ Cardanol · Heterogeneous process $\cdot$ Energy reduction $\cdot$ Solvent reduction
Abbreviations
DS Degree of substitution
AGU Anhydroglucose unit
CDA Cellulose diacetate
PA 3-Pentadecylphenoxy acetyl
PAA 3-Pentadecylphenoxy acetic acid
PLA Polylactic acid
PBS Polybutylene succinate
PBSA Polybutylene succinate adipate
GF Glass fiber
MFR Melt flow rate
DSC Differential scanning calorimetry
NMR Nuclear magnetic resonance
IR Infrared
SEM Scanning electron microscope
DMSO Dimethyl sulfoxide 
DMAP $N, N$-Dimethyl-4-aminopyridine

DMAc $N, N$-Dimethylacetamide

$\mathrm{LiCl}$ Lithium chloride

IPA Isopropyl alcohol

\section{Introduction}

In the face of petroleum resource depletion and global warming, biomass-based plastics (bioplastics), made from plant resources, which are renewable and fixate carbon dioxide, have attracted increasing attention in recent years. At present, mass-produced bioplastics, such as polylactic acid (PLA) (Li et al. 2010, Serizawa et al. 2006), use starch, which is generally produced from the edible parts of plants. Concerns over future food shortages, however, are driving efforts to use plant resources that are not edible.

We have produced novel bioplastics for use in durable products by mainly using two readily procurable inedible plant resources: cellulose, the main component of most plants and the most abundant nonfood plant resource produced on the ground, and cardanol, a uniquely structured phenol derivative with a linear alkyl side chain, derived from cashew nut shells generated in large amounts as a byproduct.

Cellulose is a polysaccharide consisting of Dglucose units linked together by $\beta 1-4$ glycosidic bonds into linear chains. It has an extensive hydrogenbonded and partially crystallized structure that makes it very stiff, non-melt processable, and insoluble in water and most organic solvents. Esterification is commonly used to produce thermoplastics from cellulose, mainly by using short chain acids such as acetic acid, propionic acid, and nitric acid (Zepnik et al. 2010). Because of these cellulose derivatives' narrow process windows between the melting and degradation temperatures, large amounts of external plasticizers, such as glycerin, polyethylene glycol, and triethyl citrate, generally must be added (Hwan-Man et al. 2004). However, adding external plasticizers can reduce the flexural strength and heat resistance of cellulose ester composites.

To overcome this problem, a number of studies have been conducted on modifying cellulose and its derivatives by bonding them with long chain alkyl components or by grafting various polymers with them. The bonding and grafting agents serve as internal plasticizers and provide additional properties. Several studies investigated the use of cellulose acetates, which are soluble in several common organic solvents, as a precursor in the grafting of various linear polymers (Roy et al. 2009), including $\varepsilon$-caprolactoneL-lactide copolymers (Nie et al. 1994), PLA (Yoshioka et al.1999, Teramoto and Nishio 2003), and styrenemaleic anhydride random copolymers (Teramoto and Nishio 2004). Other studies have investigated the use of pure cellulose as a precursor by using special solvents such as $\mathrm{N}, \mathrm{N}$-dimethylacetamide/lithium chloride (DMAc/LiCl) and ion liquids such as 1-allyl-3methylimidazolium chloride (Edgar et al. 1998) to dissolve cellulose. In these solvents, long chain alkanoic acids (carbon number: 12-20) were bonded (Sealey et al. 1996), and also graft copolymerization was conducted using various monomers such as lactic acid (Yan et al. 2009) and methacrylate (EnomotoRogers et al. 2009). However, the cellulose-based bioplastics produced in these studies did not exhibit sufficient mechanical strength, heat resistance, and water resistance for practical use in various durable products. Moreover, the bonding and grafting agents used were made from petroleum products or starch.

Cardanol is a principal organic ingredient (about $30 \mathrm{wt} \%$ ) in cashew nut shells (Kumar et al. 2002; Lubi and Thachil 2000). Cashew nut is a renewable agricultural resource, and cashew trees (Anacardium occidentale L.) are widely planted in tropical countries such as India and Vietnam. In processing cashew nuts, large quantities of shells are generated as an inedible byproduct. Cardanol is a phenol derivative consisting of a linear unsaturated hydrocarbon as a flexible and hydrophobic long side chain (carbon number: 15) and phenol as a rigid aromatic part with a reactive hydroxyl group.

The cellulose-based thermoplastic we developed is mainly made of cellulose diacetate (CDA) and a modified cardanol (3-pentadecylphenoxy acetic acid: PAA) (Iji et al. 2011, 2013). Cardanol is modified to enhance its reactivity with CDA. The unsaturated bonds in its alkyl side chain are changed to saturated ones by hydrogenation and the phenolic hydroxyl group is changed to an acetyl chloride group. Esterification of CDA with the resulting PAA chloride results in a thermoplastic, cardanol-bonded cellulose resin (PAA-bonded CDA) (Fig. 1) with high tenacity (long elongation while maintaining maximum bending 


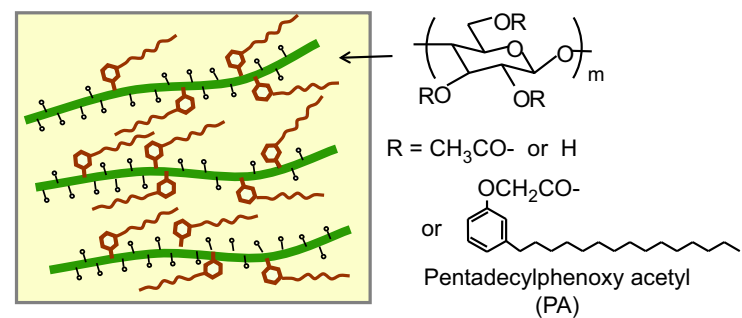

Fig. 1 Molecular structure and schematic representation of cardanol-bonded cellulose resin

strength), heat resistance, and water resistance. All of these properties are considerably better than those of a conventional CDA composite consisting of CDA and a conventional external plasticizer.

However, as PAA-bonded CDA is synthesized in a homogeneous process, in which both the starting material (CDA) and product are dissolved in a solvent, a large amount of a poor solvent is required to isolate the product, causing a large quantity of energy for production. The poor solvent is also needed to remove the remaining reactant of PAA from the product by precipitation because the reactant cannot be separated by distillation with low energy use due to its high boiling point. To cut back on poor solvents, another process using isocyanate-modified cardanol was studied (Tanaka et al. 2013). In this process, isocyanatemodified cardanol is bonded to CDA dissolved in a solvent. While the resulting resin is also dissolved in the solvent, it can be recovered without poor solvent only by evaporating the solvent because the remaining reactant coagulates and is easily removed by filtration. However, the starting material is still CDA, which is synthesized in a homogeneous process and recovered with large amounts of poor solvent.

Heterogeneous processes are promising for synthesizing cellulose resins with reduced energy consumption. In a heterogeneous process, cellulose and its derivative are not dissolved but swollen in a solvent. The resulting product can be readily recovered by solid-liquid separation without poor solvents as long as the swelling of the product is limited. However, to maintain the efficiency of solid-liquid separation, that is, productivity in the recovery step, homogeneity of the product becomes low because cellulose is not sufficiently modified by side chains, which causes insufficient thermoplasticity and other properties of the products (Fig. 2, diagonal arrow). Namely, there is an incompatibility between productivity in the

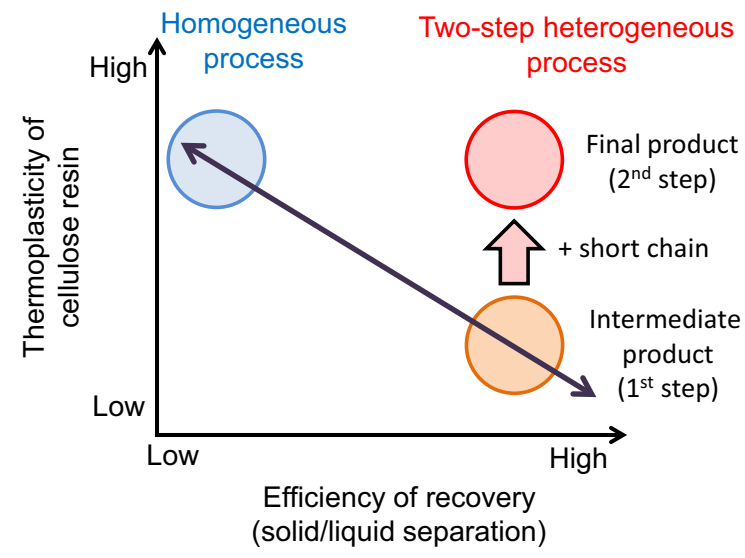

Fig. 2 Schematic representation of relationship between efficiency of recovery and thermoplasticity of cellulose resin

recovery step and thermoplasticity and other properties of a product in a usual heterogeneous process. Thermoplasticity is an index on the extent of modification or homogeneity of a resin. While many attempts have been made to synthesize cellulose esters with long side chains in heterogeneous processes, as shown in recent reviews (Edgar et al. 2001; Freire and Gandini 2006), there has been no study to resolve the incompatibility.

In this study, we developed a heterogeneous process called the "two-step heterogeneous process", to solve the above incompatibility (Toyama et al. 2014a, 2014b, Tanaka et al. 2014). Conceptual and flow diagrams of the process are shown in Figs. 2 and 3 , respectively. During the 1st step, limited amounts of the long chain (PAA) and short chain (acetic acid) components are bonded to cellulose, and the resulting intermediate products are recovered by filtration without poor solvents. Swelling of the intermediate products is controlled by bonding adequate amounts of the long and short chain components using a selected solvent and catalyst for their efficient recovery by filtration (Fig. 2, lower right circle). During the 2nd step, the short chain component is additionally bonded to the intermediate products to improve its homogeneity and, therefore, thermoplasticity (Fig. 2, upper right circle). The final product, cardanol-bonded cellulose resin, is recovered by distillation of the reaction solvent and the short chain component, which have relatively low boiling points. The solvent usage of the two-step heterogeneous process was compared with that of a homogeneous process. Main characteristics of the resultant cellulose resin for use in durable 


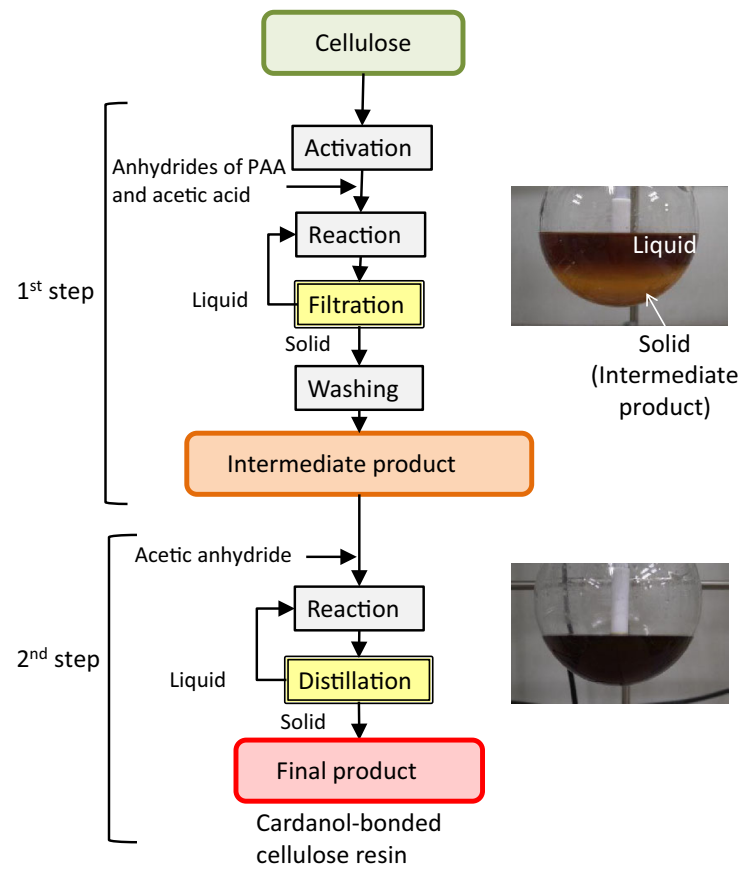

Fig. 3 Flow diagram of two-step heterogeneous process and photograph of reaction system of each step

products were investigated. Specific additives were also studied including a flexible polyester with high compatibility with the cellulose resin and glass fiber (GF) to improve the characteristics.

\section{Experimental}

\section{Materials}

Cellulose powder (KC FLOCK, W50GK) made from wood pulp was supplied by Nippon Paper Industries Co., Ltd. (Japan). The cellulose powder's diameter was $10-50 \mu \mathrm{m}$ and length was $100-500 \mu \mathrm{m}$, as we measured. Hydrogenated cardanol (m-n-pentadecyl phenol), in which the unsaturated bonds in the alkyl side chain were changed to saturated ones, was purchased from ACROS Organics (USA). The change ratio was more than $99 \%$, as measured by the company. Methanol, triethylamine, chloroform, $\mathrm{N}, \mathrm{N}$ dimethylacetamide (DMAc), lithium chloride ( $\mathrm{LiCl}$ ), 1,4-dioxane, dimethyl sulfoxide (DMSO), acetic anhydride, propionic anhydride, and pyridine were used as supplied by Kanto Chemical Co., Inc. (Japan) without further purification. 2-propanol was purchased from Tokuyama Corp. (Japan) and N,N-dimethylaminopyridine (DMAP) was purchased from SigmaAldrich Co. LLC. (USA), and these reagents were also used as received without further purification.

Polybutylene succinate (PBS, Bionolle 1001MD) and polybutylene succinate adipate (PBSA, Bionolle 3001MD) were supplied by Showa Denko K. K. (Japan). PLA (TE-4000) was produced by Unitika Ltd. (Japan). Glass fiber (03JAFT792) was supplied by Owens Corning Japan LLC.

\section{1st step of two-step heterogeneous process}

The scheme of the 1st step of our two-step heterogeneous process is shown in Fig. 4. Cellulose powder with $6 \%$ adsorbed water ( $6.0 \mathrm{~g}$ by bone-dry weight) was activated by stirring in DMSO $(90 \mathrm{ml})$ for $2 \mathrm{~h}$, and the resulting dispersion liquid was filtrated by vacuum filtration. PAA was synthesized as reported previously (Iji et al. 2013). PAA (40.2 g, 1 eq/OH) and acetic anhydride $(21.0 \mathrm{ml}, 2 \mathrm{eq} / \mathrm{OH})$ were mixed at $100{ }^{\circ} \mathrm{C}$ for an hour, resulting in a mixture of three kinds of acid anhydrides and two kinds of acids; acetic anhydride, mixed anhydride of PAA and acetic acid, anhydride of PAA, acetic acid, and PAA. Dehydrated 1,4-dioxane $(150 \mathrm{ml})$ and DMAP (3.0 g) were added to the mixture, the activated cellulose with about $13 \mathrm{~g}$ of the adsorbed DMSO was then dispersed, and the resultant dispersion liquid was stirred for $15 \mathrm{~h}$ under a dry nitrogen atmosphere. After it was cooled to room temperature, the dispersion liquid was filtrated by vacuum filtration. The filtration residue was washed three times with $440 \mathrm{ml}$ of 2-propanol at $60{ }^{\circ} \mathrm{C}$ and dried in vacuum for $5 \mathrm{~h}$ at $105{ }^{\circ} \mathrm{C}$ to obtain about $8 \mathrm{~g}$ of the intermediate product $\left(D S_{P A}=0.23-0.30\right.$, $D S_{A c}=0.9-1.1$, yield about $76 \mathrm{wt} \%$ ). Here, $D S_{P A}$ and $D S_{A c}$ represent the degree of substitution (DS) of the hydroxyl group in one anhydroglucose unit (AGU) with 3-pentadecylphenoxy acetyl (PA) and acetyl groups, respectively. The method described above was used to prepare other products with different DS values by changing the amount of PAA and acetic anhydride.

The filtrate was reused twice as follows. After adding PAA ( $70 \%$ of that for initial reaction), acetic anhydride (70 \%), dioxane (9\%), and DMAP (7\%) to the filtrate of the initial reaction, the second reaction was conducted. The third reaction was also conducted after adding the same amount of reagents to the filtrate 


$$
\begin{aligned}
& \text { 3-Pentadecylphenoxy acetic } \\
& \text { acid (PAA) }
\end{aligned}
$$

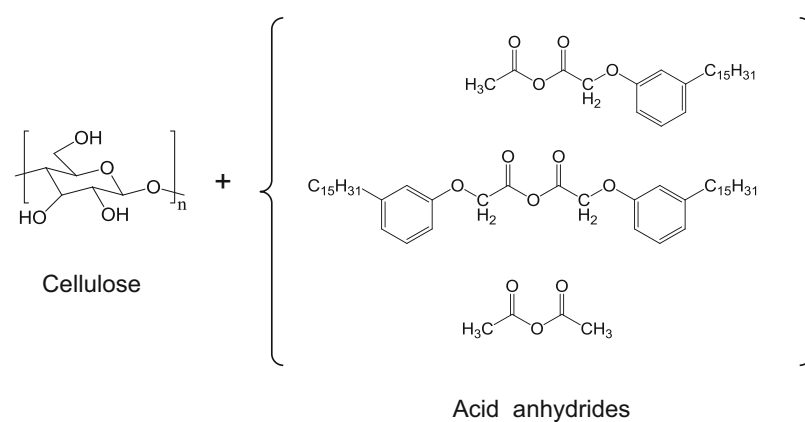

Acid anhydrides

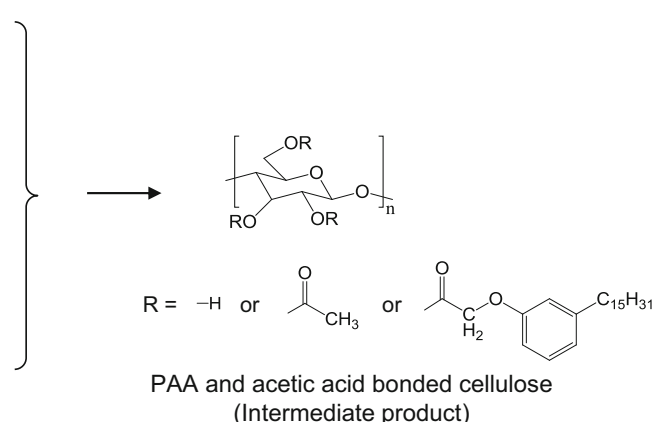

Fig. 4 Scheme of 1st step of two-step heterogeneous process

as with the second reaction. The reaction time was $3 \mathrm{~h}$ as for all these three reactions.

\section{2nd step of two-step heterogeneous process}

The intermediate product $(8.0 \mathrm{~g})$ was dispersed in a mixture of dehydrated pyridine $(54 \mathrm{ml})$ and acetic anhydride $(63.1 \mathrm{ml}, 8 \mathrm{eq} / \mathrm{OH})$, and the resultant dispersion liquid was stirred for $18 \mathrm{~h}$ at $100{ }^{\circ} \mathrm{C}$. After the reaction, the liquid components, namely, pyridine, the remaining acetic anhydride, and by-product acetic acid, were distilled for about $1.5 \mathrm{~h}$ at $100{ }^{\circ} \mathrm{C}$ under a reduced pressure of 100 Torr. The residual solid component was pulverized, dried, and the final product was obtained. After drying for an hour at $100{ }^{\circ} \mathrm{C}$, the remaining pyridine in the product was $<0.1 \mathrm{wt} \%$, as determined using a high-performance liquid chromatography system (LC-20AP system, Shimadzu Corp., Japan). For measuring physical properties, DMAP was used as a catalyst for the acetylation reaction to enhance the efficiency of the experiments by reducing the reaction time. In this case, the resulting products were precipitated and recovered using excess methanol then washed with 2-propanol to remove DMAP. All the samples were finally dried at $105{ }^{\circ} \mathrm{C}$ in vacuum for $5 \mathrm{~h}$ to accurately measure the properties of the samples. The DS values of the final product used for composites were
$D S_{P A}=0.24-0.29, D S_{A c}=2.4-2.6$ unless otherwise noted.

Homogeneous process using cellulose as starting material

By using cellulose as a starting material and dissolving it in a solvent, cardanol-bonded cellulose resins were synthesized as follows.

First, cellulose was esterified with PAA after it was dissolved in a solvent. Cellulose (7.0 g) was dispersed in a solution of DMAc $(350 \mathrm{ml})$ and $\mathrm{LiCl}(16.8 \mathrm{~g})$. The mixture was stirred at $160{ }^{\circ} \mathrm{C}$ for $2 \mathrm{~h}$ then cooled to room temperature by natural cooling, leading to dissolution of cellulose. Then triethylamine $(9.1 \mathrm{ml}$, $0.5 \mathrm{eq} / \mathrm{OH})$ and a solution of PAA chloride (16.45 g, $0.3 \mathrm{eq} / \mathrm{OH})$ in DMAc $(350 \mathrm{ml})$ were added to the solution of cellulose. PAA chloride was synthesized as reported previously (Iji et al. 2013). The resulting reaction solution was stirred at $120^{\circ} \mathrm{C}$ for $4 \mathrm{~h}$, then precipitation was conducted by adding a mixture of chloroform $(470 \mathrm{ml})$ and methanol $(930 \mathrm{ml})$. The resultant precipitate was washed with another mixture of chloroform $(470 \mathrm{ml})$ and methanol $(930 \mathrm{ml})$. The precipitate was dried in vacuum at $80{ }^{\circ} \mathrm{C}$ for $2 \mathrm{~h}$ then at $105{ }^{\circ} \mathrm{C}$ for $2 \mathrm{~h}$, resulting in $11.7 \mathrm{~g}$ of PAA-bonded cellulose. Regarding the required amount of solvents, we estimate that the amounts of reaction solvent 
(DMAc) and poor solvent (chloroform and methanol) can be reduced to 600 and $980 \mathrm{ml}$, respectively, and the amount of washing solvent can be reduced to the same amount as that used in the 1st step of the two-step heterogeneous process.

Second, the PAA-bonded cellulose was acetylated after it was dissolved in a solvent. The PAA-bonded cellulose $(4.2 \mathrm{~g})$ was pulverized and dissolved in a DMAc/LiCl solution: it was dispersed in a solution of DMAc $(530 \mathrm{ml})$ and $\mathrm{LiCl}(2.3 \mathrm{~g})$ and the mixture was stirred at $120{ }^{\circ} \mathrm{C}$ for $2 \mathrm{~h}$. The solution was then cooled to $70{ }^{\circ} \mathrm{C}$ and pyridine $(60 \mathrm{ml})$ and acetic anhydride $(84 \mathrm{ml}, 15 \mathrm{eq} / \mathrm{OH})$ were added. After the solution was stirred at $70{ }^{\circ} \mathrm{C}$ for $5 \mathrm{~h}$, precipitation was conducted by adding excess water $(1400 \mathrm{ml})$. The resultant precipitate was washed with water $(1800 \mathrm{ml})$ and methanol $(700 \mathrm{ml})$. The precipitate, cardanol-bonded cellulose resin, was dried in vacuum at $70{ }^{\circ} \mathrm{C}$ for $1 \mathrm{~h}$ then at $105{ }^{\circ} \mathrm{C}$ for $4 \mathrm{~h}$. Finally, cardanol-bonded cellulose resin of $D S_{P A}=0.18$ and $D S_{A c}=2.3$ was obtained. In terms of the required amount of the solvents, we consider that this step of acetylation can be substituted with the 2nd step of the two-step heterogeneous process to reduce solvent usage. The amount of reaction solvent can then be reduced to the same amount as that of the 2nd step, and the poor solvent and washing solvent can be eliminated.

\section{Homogeneous process using CDA as starting} material

Using CDA as a starting material and dissolving it in 1,4-dioxane, cardanol-bonded cellulose resin (PAAbonded CDA) was synthesized, as reported previously (Tanaka et al. 2013). PAA chloride was used as a PAA reactant and methanol was used as poor and washing solvents. To produce $10 \mathrm{~g}$ of PAA-bonded CDA, $152 \mathrm{~g}$ of dioxane and at least $760 \mathrm{~g}$ of methanol were required. As the product was washed with excess solvent (methanol) in this case, the amount of washing solvent in this homogeneous process was aligned to that of the two-step heterogeneous process to fairly compare solvent usage between the homogeneous and two-step heterogeneous processes.

\section{Measurements}

Sample composites were prepared by mechanically kneading the cellulose derivatives and $10-50 \mathrm{wt} \%$ of the polyester resins and GF using an extruder (HAAKE MiniLab, Thermo Fisher Scientific Inc., USA) at $210-220{ }^{\circ} \mathrm{C}$. These composites were then molded into test pieces by using an injection-molding machine (HAAKE MiniJet II, Thermo Fisher Scientific Inc., USA) operating at $210-230{ }^{\circ} \mathrm{C}$. The test pieces were $2.4 \mathrm{~mm}$ thick, $80 \mathrm{~mm}$ long, and $12.4 \mathrm{~mm}$ wide. The mechanical characteristics of the test pieces were measured by a bending test in accordance with ASTM D790 by using a bending test instrument (INSTRON 5567, Instron Co., USA). The Izod-impact test was also conducted in accordance with JIS K7110 using an impact test instrument (Universal Impact Tester C1, Toyo Seiki CO., Japan). The fracture surfaces of the test pieces after the impact test were observed with a scanning electron microscope (SEM) (VE-7800, KEYENCE Corp., Japan).

Differential scanning calorimetry (DSC) analysis was conducted using a differential scanning calorimeter (DSC 6200/EXSTAR6000, Seiko Instrument Inc., Japan). Samples were first cooled to $-100{ }^{\circ} \mathrm{C}$ then heated at a scanning rate of $10{ }^{\circ} \mathrm{C} / \mathrm{min}$, kept at $230{ }^{\circ} \mathrm{C}$ for $3 \mathrm{~min}$ (first heating scan), and immediately quenched to $-100{ }^{\circ} \mathrm{C}$ at a rate of $-50{ }^{\circ} \mathrm{C} / \mathrm{min}$. The second heating scans were run from -100 to $270{ }^{\circ} \mathrm{C}$ at a rate of $10^{\circ} \mathrm{C} / \mathrm{min}$. The heat of fusion of the crystallized polyesters was obtained from the data of the first run. The glass transition temperatures were obtained from the second run and corresponded to the midpoint of discontinuity in the heat flow.

The melt flow rate (MFR), which is the weight of a melted sample passing through the capillary (size: $10 \mathrm{~mm} \times 2 \mathrm{~mm} \varphi$ ) in $10 \mathrm{~min}$, was estimated using a capillary rheometer (CFT-500D, Shimadzu Co., Japan) at $200{ }^{\circ} \mathrm{C}$ with a $500-\mathrm{kgf} / \mathrm{cm}^{2}$ load. Each sample was kept for $5 \mathrm{~h}$ in a $105{ }^{\circ} \mathrm{C}$ preheated oven before the MFR test to remove humidity.

Water resistance was evaluated by calculating the water absorption ratios of the test pieces by weighing them before and after soaking them for $24 \mathrm{~h}$ in distilled water at room temperature.

DS calculation

The DS values of the products synthesized in the heterogeneous process were determined through ${ }^{1} \mathrm{H}$ NMR as for representative samples. The products were propionylated before NMR spectroscopy as reported previously (Tezuka and Tsuchiya 1995) to 
make them soluble in deuterated chloroform. This propionylation is considered not to affect the PA and acetyl groups as the DS values did not change even when the propionylation was conducted for longer duration with excess propionic anhydride. An example of NMR spectrum is shown in Fig. 6 in the "Results and discussion" section. The DS values were calculated from the NMR spectra as follows.

$D S_{P A}=\frac{7 \times I_{\delta 0.86}}{\left(3 \times I_{\delta 3.0-5.5}-2 \times I_{\delta 0.86}\right)}$

$D S_{A c}=D S_{P A A} \times \frac{I_{\delta 1.8-2.2}}{I_{\delta 0.86}}$

where $\mathrm{I}_{\delta 0.86}$ denotes the integrals of the triplet peak at $\delta 0.86$ assigned to the protons of terminal methyl group in the PA group, $\mathrm{I}_{\delta 3.0-5.5}$ denotes the integrals of the multiple peaks from $\delta 3.0$ to $\delta 5.5$ including the protons of AGU and the methylene protons next to the carbonyl group in the PA group, and $\mathrm{I}_{\delta 1.8-2.2}$ denotes the integrals of the multiple peaks from $\delta 1.8$ to $\delta 2.2$ assigned to the methyl protons in the acetyl group.

On the other hand, the heights of specific peaks of infrared (IR) absorbance spectra are well correlated with the DS values evaluated through NMR spectroscopy as shown in Fig. 5 (Matsumura et al. 2000). Therefore, the DS values were also evaluated through IR spectroscopy to enhance the efficiency of the experiments regarding the products of solvent distillation experiments at the 2nd step, solvent reuse experiments, and those used for composites. IR spectra were measured using a spectrometer (FTIR-4100, Jasco Corp., Japan). An example of IR spectrum is shown in Fig. 7. The $D S_{P A}$ was calculated using the signal of the aromatic ring at $1586 \mathrm{~cm}^{-1}$ and the total DS $\left(D S_{\text {total }}\right)$ of the PA and acetyl groups was calculated using that of carbonyl stretching around
$1750 \mathrm{~cm}^{-1}$. The heights of these peaks were normalized by the peak height of the ether stretching of AGU around $1050 \mathrm{~cm}^{-1}$. To determine the calibration curve for $D S_{P A}$ (Fig. 5), PAA-bonded CDAs with different $D S_{P A}$ were used, which were synthesized in the homogeneous process described above by changing the amount of the reactant of PAA. For $D S_{\text {total }}$, the products synthesized in the two-step heterogeneous process with different $D S_{\text {total }}$ were used: the same intermediate product of the 1st step was used as the starting material, and the reaction time at the 2nd step was changed to obtain the products with different $D S_{\text {total }}$.

\section{Results and discussion}

Results of 1st step of two-step heterogeneous process

In the 1st step of the two-step heterogeneous process, cellulose was esterified with limited amounts of PAA as the long chain component and acetic acid as the short chain component in a heterogeneous system using dioxane as a reaction solvent and DMAP as a catalyst. The resultant intermediate products containing PA and acetyl groups at cellulose chains were adequately swollen in the solvent then recovered by filtration without poor solvents.

The intermediate products were characterized through ${ }^{1} \mathrm{H}$ NMR and IR spectroscopy. Figure 6 shows an NMR spectrum of a propionylated intermediate product. The DS values of the PA and acetyl groups were calculated using the integrals of the methyl protons of the PA and acetyl groups $\left(D S_{P A}=0.23\right.$ and $\left.D S_{A c}=0.90\right)$. Figure 7 shows the IR absorption spectrum of the same intermediate
Fig. 5 Calibration curves for determining DS values from IR spectra: a long side chain (PA group) and b short side chain (acetyl group)
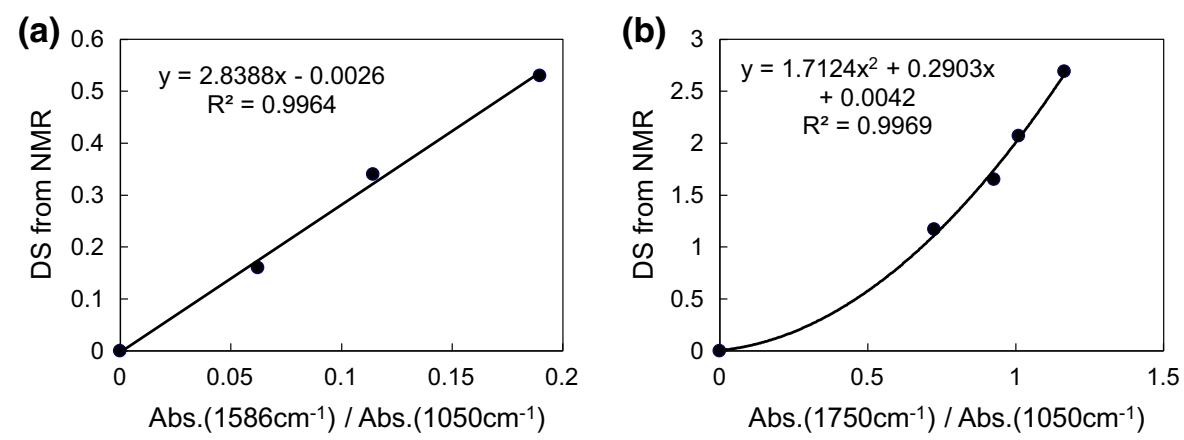
Fig. 6 NMR spectrum of propionylated intermediate product of two-step heterogeneous process
Fig. 7 IR spectrum of intermediate product of twostep heterogeneous process
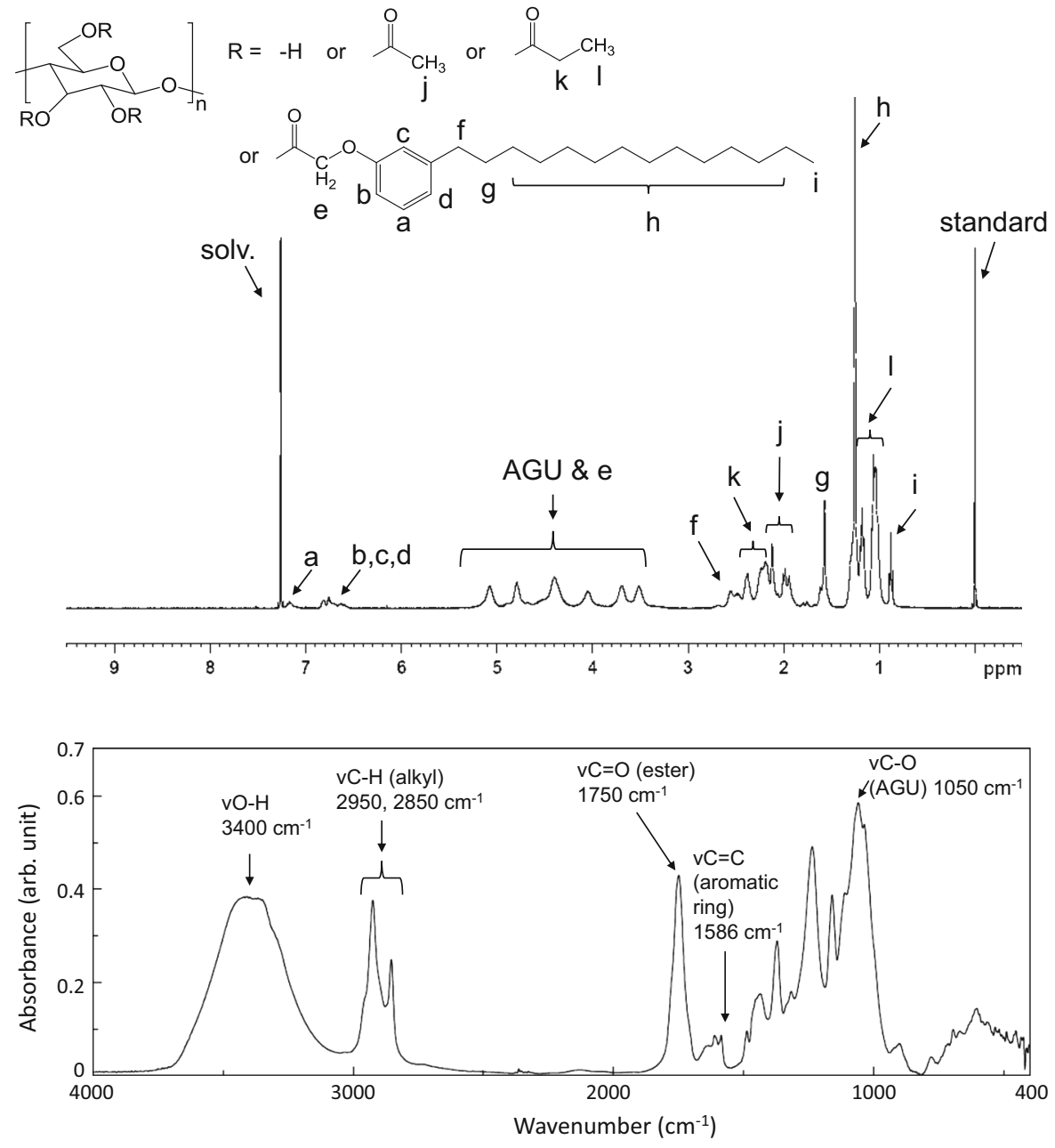

product. The formation of the ester bond is confirmed by the $\mathrm{C}=\mathrm{O}$ stretching peak around $1750 \mathrm{~cm}^{-1}$. Bonding of PAA is shown by the peaks originating from the skeletal stretching of the aromatic ring $\left(1586 \mathrm{~cm}^{-1}\right)$ and stretching of the $\mathrm{C}-\mathrm{H}$ bonds of the alkyl chain $\left(2850,2950 \mathrm{~cm}^{-1}\right)$.

Suitable DS values of the PA and acetyl groups were achieved effectively by selecting solvents and catalysts. We investigated reaction solvents such as ether, amide, amine, ester, and ketone. With ethers, including dioxane, effective reaction, as well as good efficiency of solid-liquid separation, were achieved. For amide and amine, the intermediate products tended to swell too much, causing difficulty in recovery. For ester and ketone, the products tended to have a relatively small amount of the long chain component. Regarding catalysts, acid catalysts were not adopted to avoid a decrease in the molecular weight of the cellulose resins and to achieve high mechanical strength. We adopted DMAP because it exhibits high catalytic ability among basic catalysts, which enables effective reaction of PAA, as explained below.

We investigated the relationship between the thermoplasticity of the resulting intermediate products and the productivity of its recovery using the abovementioned solvent and catalyst. In Fig. 8, the thermoplasticity (MFR) of the intermediate products is shown against the solid/liquid ratio of the filtration residue, which is a measure of efficiency in the solid-liquid separation, namely, productivity of the recovery step. The DS values of the products are shown in brackets. While the solid/liquid ratio increased as the DS values of the PA and acetyl groups decreased, the MFR decreased at the same time, which demonstrates an incompatibility between the thermoplasticity of the 


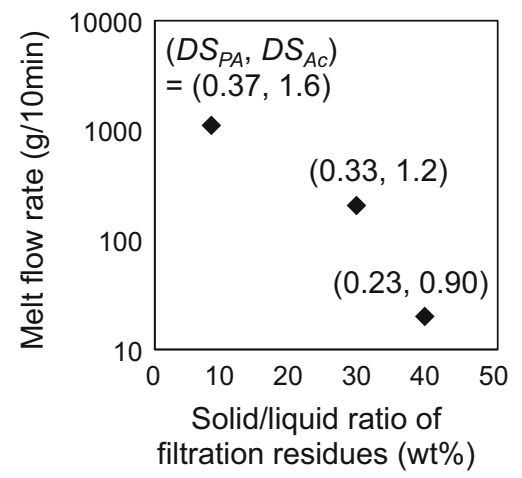

Fig. 8 Relationship between melt flow rate of intermediate products (cellulose with PA and acetyl groups) and solid/liquid ratio of their filtration residue at 1 st step of two-step heterogeneous process

intermediate products and their productivity, as illustrated in Fig. 2.

The increase in the solid/liquid ratio is attributed to the suppressed swelling of the intermediate products in the reaction solvent. The less cellulose is modified, the lower affinity its derivative has for most organic solvents owing to strong intra and intermolecular hydrogen bonding between residual hydroxyl groups in the derivative. The decrease in thermoplasticity is explained by the strong hydrogen bonding and limited plasticizing effect of the long chain component due to its limited bonding amounts.

As shown in Fig. 8, when $D S_{P A}$ was $<0.33$ and $D S_{A c}$ was $<1.2$, the intermediate products exhibited good efficiency of solid-liquid separation (solid/liquid ratio of filtration residue $>30 \%$ ). On the other hand, we found out that when $D S_{P A}$ was more than 0.2 and $D S_{A c}$ was more than or equal to 0.9 , the final products exhibited sufficient thermoplasticity and other properties after the 2nd step of the two-step heterogeneous process, as discussed later. In summary, by controlling the DS values $\left(D S_{P A}=0.2-0.3\right.$ and $\left.D S_{A c}=0.9-1.2\right)$ we achieved high efficiency in the solid-liquid separation of the intermediate products as well as sufficient thermoplasticity and other properties of the final product (resulting resin) after the second step.

Figure 9 shows the DS values of the long and short chain components of the intermediate products as a function of the reaction time. The DS values sufficient for thermoplasticity of the final product $\left(D S_{P A}>0.2\right)$ were achieved after reaction of at least $3 \mathrm{~h}$, exhibiting effective bonding of the long chain component (PAA).
Considering the steric hindrance of PAA, which contains a long chain part of 15 carbons and a aromatic ring, we can say that relatively high $D S_{P A}$ values were achieved in a competing reaction of PAA and acetic acid $\left(D S_{P A} / D S_{A c}\right.$ about 1/4). This is attributed to the following two reasons. First, swelling of the cellulose and cellulose derivatives by the solvent, dioxane, is thought to help the reactant of PAA penetrate into the cellulose and its derivatives. Second, the basic catalyst, DMAP, is supposed to generate a reactive intermediate of PAA more effectively than that of acetic acid because the carbonyl group of PAA is more electrophilic; thus, it tends to be attacked more by a catalyst compared with the carbonyl group of acetic acid. This electrophilicity is due to the electron-withdrawing inductive effect of the oxygen atom next to the alpha carbon.

We also confirmed that the recovered solvent and the remaining long and short chain components can be reused, as discussed later.

Results of 2nd step of two-step heterogeneous process

The intermediate products of the 1st step were additionally bonded with the short chain component in the 2nd step. The resulting final product, cardanolbonded cellulose resin, was recovered without poor solvents. It was recovered only by distilling the solvent (pyridine) and the remaining short chain component, which exhibit relatively low boiling points.

The thermoplasticity of the intermediate products increased by the additional bonding of the short chain component in the 2nd step. Figure 10 illustrates the relationship between the MFR of both the intermediate and final products of the two-step heterogeneous process and the weight ratio of the side chains (the long and short chain components) in these products. This weight ratio represents the extent of the plasticizing effect of the side chains. Although the intermediate products $\left(D S_{A c}\right.$ about 0.9$)$ exhibited low thermoplasticity (MFR $=10-20 \mathrm{~g} / 10 \mathrm{~min}$ ) and were not sufficient for injection molding, after additional bonding of the short chain component at the 2nd step, the final products $\left(D S_{A c}\right.$ about 2.4) exhibited high thermoplasticity (MFR $=300-900 \mathrm{~g} / 10 \mathrm{~min}$ ) and were sufficiently applicable for injection molding. It was also found that the amount of $D S_{P A}$, about 0.25 , was sufficient to obtain cardanol-bonded cellulose 
Fig. 9 DS values of a long side chain (PA group) and b short side chain (acetyl group) of resin as function of reaction time in 1st step of two-step heterogeneous process

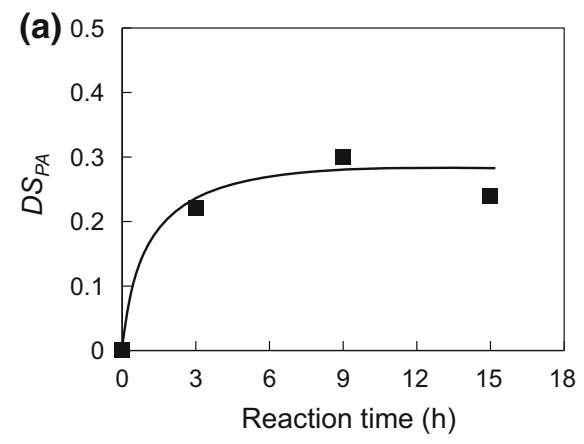

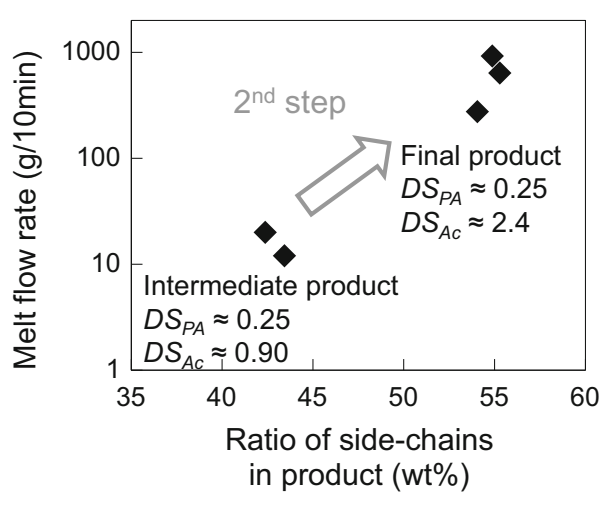

Fig. 10 Melt flow rates of intermediate and final products of two-step heterogeneous process

resins with sufficient thermoplasticity for injection molding.

\section{Comparison of productivity between two-step}

heterogeneous process and homogeneous process

We compared the solvent usage of the two-step heterogeneous process and that of a conventional homogeneous process to compare their productivity. Solvent usage represents the energy consumption of a process because the energy used for heating and distillation of solvents constitutes a large fraction of the energy consumed in the process.

Before the comparison, we reduced the solvent usage in the procedure of the two-step heterogeneous process described in the Experimental section because excess solvents had been used with the aim of accurately measuring the product properties. We reduced the reaction solvent (dioxane) and washing solvent (isopropyl alcohol: IPA) in the 1st step, and the reaction solvent (pyridine) in the 2 nd step. First, dioxane was reduced by $60 \%$ by reusing it twice. The

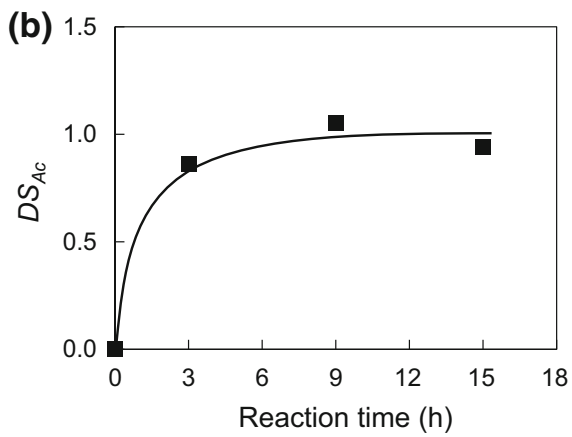

$D S_{P A}$ values of the resultant products of the second and third reactions were over $70 \%$ that of the initial reaction, which we assume is sufficient for actual use because the amount of supplementary PAA (70 \% of the initial reaction) can be further increased. Second, IPA was reduced by $93 \%$ by reusing it twice and cutting back the amount of IPA by $80 \%$ in each washing step. The remaining unreacted PAA could be reduced to $<1 \mathrm{wt} \%$ of the product by this washing. Third, the amount of both pyridine and acetic anhydride was halved in the 2 nd step. It was found that although the reaction speed became about $1 / 2$, the same level of the $D S_{A c}$ value as the original case could be obtained.

The solvent usage of the heterogeneous process after the solvent reduction is summarized in Table 1 as well as that of the homogeneous process. For the homogeneous process, we chose the process using $\mathrm{CDA}$ as a starting material. Although we attempted synthesis of cardanol-bonded cellulose resin using cellulose as a starting material and dissolving it in an ordinary solvent for cellulose $(\mathrm{DMAc} / \mathrm{LiCl})$, gelation of the product limited $D S_{P A}$; therefore, its thermoplasticity was too low for injection molding. The gelation occurred when the concentration of the PAA reactant or cellulose was high. The reason of the gelation is considered to be due to the hydrophobic interaction of the PA groups, which is highly hydrophobic compared with cellulose because of its long alkyl chain and aromatic ring. While we attempted simultaneous bonding of PAA and acetic acid to make cellulose more hydrophobic and more soluble in the solvent by acetylation, the gelation still occurred. Since gelation did not occur when we used CDA as a starting material, we eventually adopted a process using CDA. In the process using cellulose as a starting material, the required amount of solvent used at each 
step is estimated to be $407 \mathrm{~g}$ for reaction, $723 \mathrm{~g}$ for precipitation, $73 \mathrm{~g}$ for washing, and the total solvent usage is estimated to be $1203 \mathrm{~g}$ to produce $10 \mathrm{~g}$ of the resin. If the concentration of cellulose in the solvent was greatly reduced, the gelation would be avoided, resulting in obtaining the resin with higher thermoplasticity due to the increase in $D S_{P A}$. However, much more amounts of solvents will be required in this case.

The solvent usage of the two-step heterogeneous process was lower by at least $83 \%$ than that of the homogeneous process to bond PAA to CDA (Table 1). We do not include the solvent usage to produce CDA. The elimination of the poor solvent mainly contributed to the solvent reduction. Regarding the process to produce CDA, the amount of reaction solvent, which is the sum of initial solvent and the solvents added for the hydrolysis step of cellulose triacetate to obtain CDA, was estimated to be $29 \mathrm{~g}$ and that of a poor solvent was estimated to be $121 \mathrm{~g}$ per $10 \mathrm{~g}$ of the cardanol-bonded cellulose resin (Bogan and Brewer 1985). Washing solvent (water) was excluded because its reuse by distillation can be avoided: thus, its influence on the energy consumption is considered to be limited. Combined with the data in Table 1, we estimated that the solvent usage of the overall homogeneous process is $1135 \mathrm{~g}$, and it can be reduced by $86 \%$ with the two-step heterogeneous process. We believe that our two-step heterogeneous process requires energy consumption of about $1 / 10$ that of the homogeneous process.

Characteristics of cardanol-bonded cellulose resins of heterogeneous process and their composites

We evaluated the characteristics of the cardanolbonded cellulose resins produced in the two-step heterogeneous process and their composites: mechanical properties (maximum flexural strength, elastic modulus, breaking strain, impact strength), heat resistance (glass transition temperature), thermoplasticity (MFR), and water resistance (water absorption ratio), which are essential properties for use in durable products. Table 2 lists the characteristics of the resin synthesized in the heterogeneous process as well as those of PAA-bonded CDA $\left(D S_{P A}=0.33\right.$, $\left.D S_{A c}=2.1\right)$ synthesized in a conventional homogeneous process and the composites of these resins.

The cardanol-bonded cellulose resin of the heterogeneous process was found to show higher thermoplasticity, elastic modulus, and water resistivity compared with the PAA-bonded CDA of the homogeneous process. Moreover, the heat resistivity (glass transition temperature) was very close between these resins. On the other hand, the resin of the heterogeneous process was found to be brittle: they showed lower impact strength, flexural strength, and breaking strain compared with PAA-bonded CDA. Figure 11a shows the fracture cross-section of a test piece of the product after the impact strength test. Observation of the cross-section revealed the presence of voids and cellulose fiber-like substances, denoted with arrows, which would have been the origin of the fracture. We argue that such parts are generated due to insufficient bonding of the long and short chain components to cellulose, and such inhomogeneity in the resulting resins mainly caused the resins' brittleness.

To expand the application areas of the resin by overcoming its brittleness, we additionally studied additives for the resin. For the homogeneous process, we had already found that PBSA is a suitable additive for PAA-bonded CDA after comparing various aliphatic polyesters such as PBSA, PBS, and PLA (Soyama et al. 2014). PBSA exhibited the best compatibility and its flexible molecular structure resulted in improvement in the impact strength of

Table 1 Solvent usage of homogeneous and two-step heterogeneous processes to produce $10 \mathrm{~g}$ of products

\begin{tabular}{lllccc}
\hline & DS of products & Reaction solvent $(\mathrm{g})$ & Poor solvent $^{\mathrm{b}}(\mathrm{g})$ & Washing solvent $(\mathrm{g})$ Total $(\mathrm{g})$ \\
\hline $\begin{array}{l}\text { Homogeneous process } \\
{ }^{\mathrm{a}}\end{array}$ & PA: 0.30-0.35 Ac: 2.4 & 152 & 760 & $73^{\mathrm{c}}$ & 985 \\
$\begin{array}{l}\text { Two-step heterogeneous } \\
\text { process }\end{array}$ & PA: 0.25 Ac: 2.4 & $91^{\mathrm{d}}$ & 0 & 73 & 164 \\
\hline
\end{tabular}

\footnotetext{
${ }^{a}$ Process to produce PAA-bonded CDA from CDA

${ }^{b}$ For precipitation of dissolved product

c Aligned with that of two-step heterogeneous process

d 1st step: $63 \mathrm{~g}$, 2nd step: $28 \mathrm{~g}$
} 


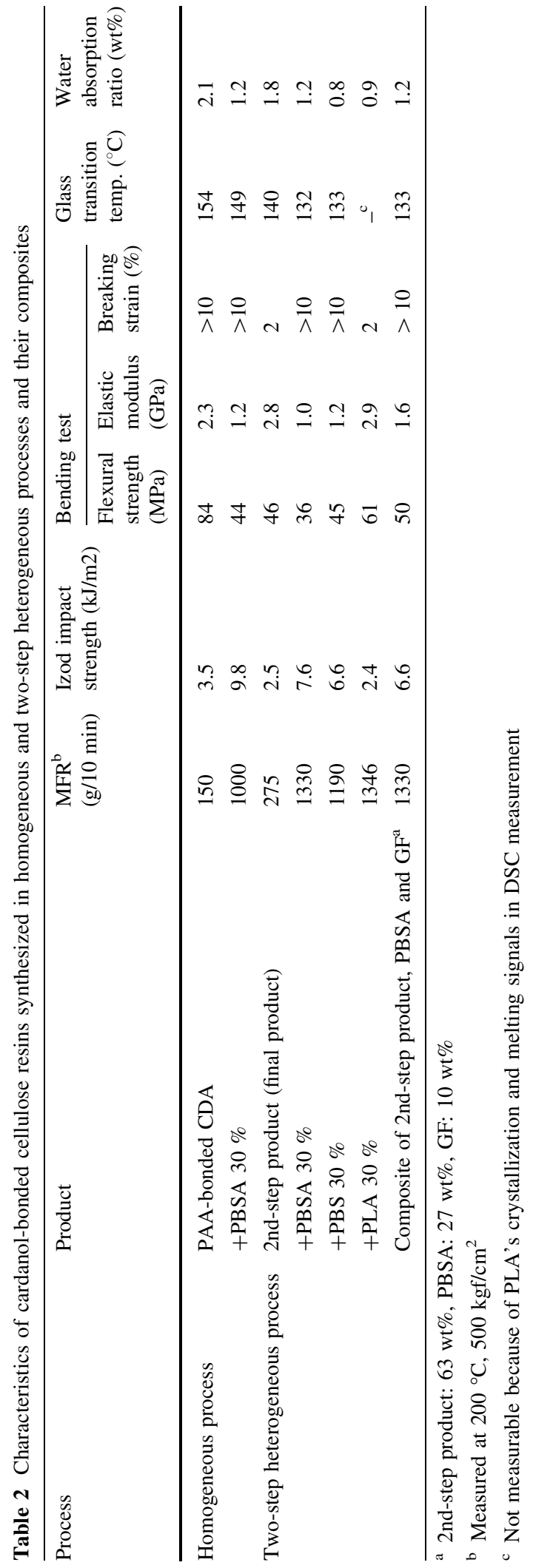

PAA-bonded CDA. For the two-step heterogeneous process, comparison of composites of the final product and $30 \mathrm{wt} \%$ of polyesters (PBSA, PBS, or PLA) revealed that PBSA also exhibited the best compatibility with this cellulosic resin. Better compatibility of PBSA and PBS than PLA was obvious from the observation that the composites with PBSA and PBS were translucent while that of PLA was more turbid. Furthermore, the compatibility of PBSA was found to be better than PBS from DSC measurements. The heat of fusion of the crystalline PBSA in the composite was $7.8 \mathrm{~J} / \mathrm{g}$, and this value was only $17 \%$ that for pure PBSA, while those values were $12.9 \mathrm{~J} / \mathrm{g}$ and $28 \%$ for PBS. The lower amount of the crystalline part indicates better compatibility of the polyester. It is believed that PBSA exhibited the best compatibility with the cardanol-bonded cellulose resin because the polarity of PBSA is the closest to the cellulose resin.

The brittleness of the resins was found to decrease by the addition of PBSA and PBS. The properties of the composites consisting of the cellulose resin and the polyester additives including PBSA, PBS and PLA are listed in Table 2. Adding PBSA and PBS improved the impact strength and breaking strain of the cellulose resin while adding PLA did not. The composite of the cellulose resin and PBSA particularly exhibited higher impact strength. This is attributed to the higher solubility of PBSA with the cellulose resin. Thus, we evaluated the composites using PBSA in detail.

Figure 11b shows the fracture cross-section of a composite with $30 \mathrm{wt} \%$ of PBSA. The voids disappeared by the addition of PBSA due to its high compatibility. At larger scales, corrugations were observed on the fracture cross-section, which demonstrates the occurrence of shearing deformation (micrograph not shown). As mentioned above, PBSA tends to form not a crystalline state but an amorphous state in the composites. Therefore, the flexible molecular structure of PBSA is expected to result in the flexibility of the composites. Due to the increased flexibility, the impact strength increased and elastic modulus decreased monotonously as the amount of PBSA increased (Fig. 12).

For comparison, PBSA was also added to the cardanol-bonded cellulose resin (PAA-bonded CDA) synthesized in a homogeneous process (Table 2). The addition of PBSA lead to similar changes in characteristics as the resin synthesized in the two-step heterogeneous process: increase in impact strength 


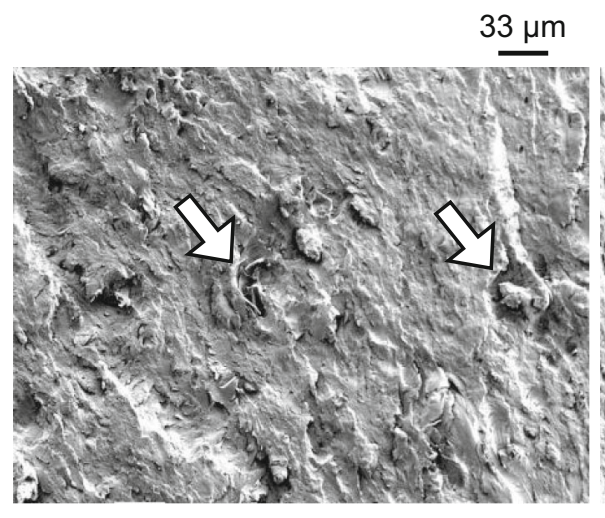

(a)

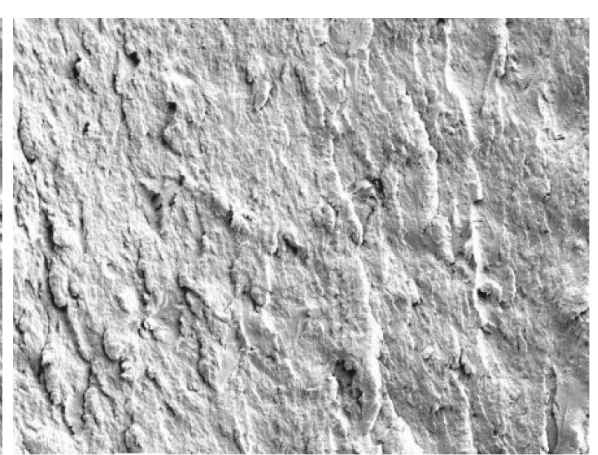

(b)

Fig. 11 SEM micrograph of fracture cross-section of a cardanol-bonded cellulose resin synthesized in two-step heterogeneous process and $\mathbf{b}$ its composite with $30 \mathrm{wt} \%$ of PBSA

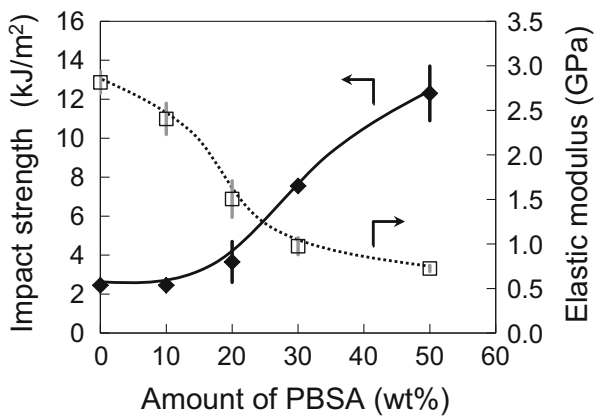

Fig. 12 Impact strength and elastic modulus of composites of cardanol-bonded cellulose resin synthesized in two-step heterogeneous process and PBSA

and decrease in flexural strength and elastic modulus. Although the composite properties of the homogeneous process were almost higher than those of the two-step heterogeneous process, the differences were small.

Since the addition of PBSA reduced the elastic modulus of the composites of the resin synthesized in the heterogeneous process, we further added GF, resulting in improvement in the elastic modulus while maintaining the impact strength (Fig. 13). As the amount of GF increased, elastic modulus increased monotonously, reaching 1.6 times $(10 \mathrm{wt} \%)$ and 2.6 times ( $25 \mathrm{wt} \%)$ of the original value. In the meantime, impact strength decreased only slightly by about $15 \%$. We argue that impact strength was maintained in spite of the addition of the rigid component due to the energy loss caused by the pullout of the GF. Indeed, there were holes generated by pulling out of

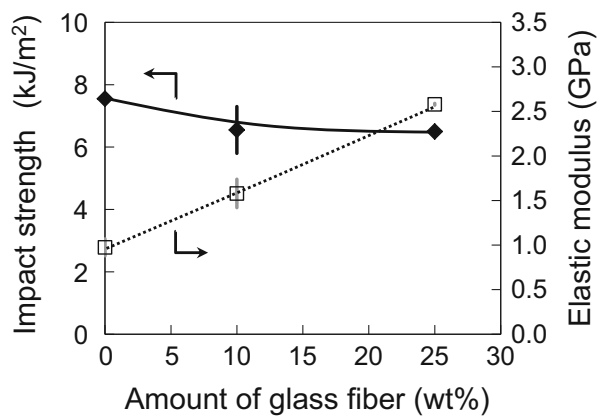

Fig. 13 Impact strength and elastic modulus of composites of cardanol-bonded cellulose resin synthesized in two-step heterogeneous process as function of amount of glass fiber. Ratio of cellulose resin and PBSA is 70:30 by weight

the GF and unbroken GF at the fracture cross-section. Finally, adding $27 \mathrm{wt} \%$ of PBSA and $10 \mathrm{wt} \%$ of GF lead to high levels of practicality in durable products as shown in Table 2.

\section{Conclusions}

A two-step heterogeneous process with low energy consumption was developed for a novel cellulosebased bioplastic with a cardanol derivative, achieving high mechanical strength and high heat resistance. The bioplastic is a cellulose ester bonded with acetic acid as a short chain component and a modified cardanol as a long chain component. In the first step, by selecting the solvent and catalyst and controlling the bonding amounts of the long and short chain components to 
cellulose, adequately swollen intermediate products are formed in the solvent. As a result, the resulting intermediate products are efficiently recovered by filtration. In the second step, the intermediate products are additionally bonded with the short chain component in another solvent to attain final products (cardanol-bonded cellulose resins), which are recovered readily only by distilling the solvent and the remaining short chain component. The total solvent usage was reduced by about $90 \%$ compared with a conventional homogeneous process using poor solvents to recover the resulting resins, which leads to a large reduction in energy. The produced cardanolbonded cellulose resins exhibited higher thermoplasticity, elastic modulus, water resistivity, and close heat resistivity compared with that of the homogeneous one. As the resin showed brittleness, further improvement in the process is desired. On the other hand, additional study on additives revealed that the brittleness can be decreased by adding specific polyesters. The mechanical and thermal characteristics of the resins were greatly improved by adding polybutylene succinate adipate and a glass fiber, reaching high target levels for durable products. We therefore conclude that the new two-step heterogeneous process is promising for mass production of novel cellulose resins and composites based on these resins are prospective for use in various durable products such as electronic devices.

Acknowledgments The authors are grateful to Ms. Toshie Miyamoto and Dr. Fumi Tanabe for their support in the experiments. The work was supported by the Japan Science and Technology Agency's Advanced Low Carbon Technology Research and Development Program (ALCA).

Open Access This article is distributed under the terms of the Creative Commons Attribution License which permits any use, distribution, and reproduction in any medium, provided the original author(s) and the source are credited.

\section{References}

Bogan RT, Brewer RJ (1985) Cellulose Esters, Organic. In: Mark HF, Bikales N, Overberger CG, Menges G, Kroschwitz JI (eds), Encyclopedia of polymer science and engineering vol 3, Wiley, New York, pp 158-181

Edgar KJ, Pecorini TJ, Glasser WG (1998) Preparation, properties, and perspective, cellulose derivatives, chapter 3: 38-60, ACS symposium series, vol 688. American Chemical Society, Washington
Edgar KJ, Buchanan CM, Debenham JS, Rundquist PA, Seiler BD, Shelton MC, Tindall D (2001) Advances in cellulose ester performance and application. Prog Polym Sci 26:1605-1688

Enomoto-Rogers Y, Kamitakahara H, Takano T, Nakatsubo F (2009) Cellulosic graft copolymer: poly(methacrylate) with cellulose side chain. Biomacromolecules 10:2110-2117

Freire CSR, Gandini A (2006) Recent advances in the controlled heterogeneous modification of cellulose for the development of novel materials. Cellulose Chem Technol 40:691-698

Hwan-Man P, Manjusri M, Lawrence TD, Mohanty AK (2004) Green nanocomposites from cellulose acetate Bioplastic and clay: effect of eco-friendly triethyl citrate plasticizer. Biomacromolecules 5:2281-2288

Iji M, Moon S, Tanaka S (2011) Hydrophobic, mechanical, and thermal characteristics of thermoplastic cellulose diacetate bonded with cardanol from cashew nut shell. Polym J 43:738-741

Iji M, Toyama K, Tanaka S (2013) Mechanical and other characteristics of cellulose ester bonded with cardanol from cashew nut shells and additional aliphatic and aromatic components. Cellulose 20:559-569

Kumar PP, Parmashivappa R, Vithayathil PJ, Rao PVS, Rao AS (2002) Process for isolation of cardanol from technical cashew (Anacardium occidentale L.) nut shell liquid. J Agric Food Chem 50:4705-4708

Li S, Ernst W, Martin P (2010) Present and future development in plastics from biomass. Biofuels Bioprod Biorefining 4(1):25-40

Lubi MC, Thachil ET (2000) Cashew nut shell liquid (CNSL) a versatile monomer for polymer synthesis. Des Monomers Polym 3(2):123-153

Matsumura H, Sugiyama J, Glasser WG (2000) Cellulosic Nanocomposites. I. Thermally deformable cellulose hexanoates from heterogeneous reaction. J Appl Polym Sci 78:2242-2253

Nie L, Narayan R (1994) Grafting cellulose acetate with styrene maleic anhydride random copolymers for improving dimensional stability. J Appl Polym Sci 54:601-617

Roy D, Semsarilar M, Guthrie JT, Perrier S (2009) Cellulose modification by polymer grafting: review. Chem Soc Rev 38:2046-2064

Sealey JE, Samaranayake G, Todd JG, Glasser WG (1996) Novel cellulose derivatives. IV. Preparation and thermal analysis of waxy esters of cellulose. J Polym Sci B Poly Phys 34:1613-1620

Serizawa S, Inoue K, Iji M (2006) Kenaf-fiber-reinforced poly(lactic acid) used for electronic products. J Appl Polym Sci 100(1):618-624

Soyama M, Iji M, Tanaka S, Toyama K (2014) Development of cardanol-bonded cellulose resin using non-food plant resources (2): improvement in practical characteristics including impact strength. Polym Prepr Jpn 63:3683-3684

Tanaka S, Honzawa H, Iji M (2013) Development of cardanolbonded cellulose thermoplastics: high productivity achieved by using isocyanate-modified cardanol. J Appl Polym Sci 130:1578-1587

Tanaka S, Toyama K, Soyama M, Iji M (2014) Development of cardanol-bonded cellulose thermoplastics: two step heterogeneous synthesis process with low energy. Preprints 
of the 21th annual meeting of the Cellulose Society of Japan: K18

Teramoto Y, Nishio Y (2003) Cellulose diacetate-graftpoly(lactic acid)s: synthesis of wide-ranging compositions and their thermal and mechanical properties. Polymer 44:2701-2709

Teramoto Y, Nishio Y (2004) Biodegrable cellulose diacetategraft-poly(L-lactide): enzymatic hydrolysis behavior and surface morphological characterization. Biomacromolecules 5:407-414

Tezuka Y, Tsuchiya Y (1995) Determination of substituent distribution in cellulose acetate by means of a ${ }^{13} \mathrm{C}$ NMR study on its propanoated derivative. Carbohydr Res 273:83-91

Toyama K, Soyama M, Tanaka S, Iji M (2014a) Development of cardanol-bonded cellulose resin using non-food plant resources: low energy heterogeneous synthesis process. Preprints of the 248th ACS national meeting: POLY 683
Toyama K, Tanaka S, Iji M (2014b) Development of cardanolbonded cellulose resin using non-food plant resources (1): low energy heterogeneous synthesis process. Polym Prepr Jpn 63:3681-3682

Yan C, Zhang J, Lv Y, Yu J, Wu J, Zhang J, He J (2009) Thermoplastic cellulose-graft-poly(L-lactide)copolymers homogeneously synthesized in an ionic liquid with 4-dimethylaminopyridine catalyst. Biomacromolecules 10(8):2013-2018

Yoshioka M, Hasegawa N, Shiraishi N (1999) Themoplastization of cellulose acetates by grafting of cyclic esters. Cellulose 6:193-212

Zepnik S, Kesselring A, Kopitzky R, Michels C (2010) Basics of cellulosics. Bioplastic Mag 5:44-47 